\title{
Bio Micro-Nano Technologies of Antioxidants Optimised Their Pharmacological and Cellular Effects, ex vivo, in Pancreatic $\beta$-Cells
}

This article was published in the following Dove Press journal:

Nanotechnology, Science and Applications

\author{
Armin Mooranian (1D' \\ Nassim Zamani' \\ Momir Mikov $\mathbb{D}^{2}$ \\ Svetlana Goločorbin-Kon ${ }^{3}$ \\ Goran Stojanovic (D) ${ }^{4}$ \\ Frank Arfuso ${ }^{5}$ \\ Bozica Kovacevic' \\ Hani Al-Salami (ID) \\ 'Biotechnology and Drug Development \\ Research Laboratory, School of Pharmacy \\ and Biomedical Sciences, Curtin Health \\ Innovation Research Institute (CHIRI), \\ Curtin University, Perth, Western \\ Australia, Australia; ${ }^{2}$ Department of \\ Pharmacology, Toxicology and Clinical \\ Pharmacology, Faculty of Medicine, \\ University of Novi Sad, Novi Sad, Serbia; \\ ${ }^{3}$ Department of Pharmacy, University of \\ Novi Sad, Novi Sad, Serbia; ${ }^{4}$ Faculty of \\ Technical Sciences, University of Novi \\ Sad, Novi Sad, Serbia; ${ }^{5}$ Stem Cell and \\ Cancer Biology Laboratory, School of \\ Pharmacy and Biomedical Sciences, \\ Curtin Health Innovation Research \\ Institute, Curtin University, Perth, \\ Western Australia, Australia
}

Introduction: Recent formulation and microencapsulation studies of probucol (PB) using the polymer sodium alginate (SA) and bile acids have shown promising results but $\mathrm{PB}$ stability, and pharmacology profiles remain suboptimal. This study aimed to investigate novel polymers for the nano and micro encapsulation of $\mathrm{PB}$, with the anti-inflammatory bile acid ursodeoxycholic acid (UDCA).

Material and methods: Six formulations using three types of polymers were investigated with and without UDCA. The polymers were NM30D, RL30D, and RS30D and they were mixed with SA and $\mathrm{PB}$ at set ratios and microencapsulated using oscillating-voltagemediated nozzle technology coupled with ionic gelation. The microcapsules were examined for physical and biological effects using pancreatic $\beta$-cells.

Results and discussion: UDCA addition did not adversely affect the morphology and physical features of the microcapsules. Despite thermal stability remaining unchanged, bile acid incorporation did enhance the electrokinetic stability of the formulation system for NM30D and RL30D polymers. Mechanical stability remained similar in all groups. Enhanced uptake of PB from the microcapsule by pancreatic $\beta$-cells was only seen with NM30D-UDCA-intercalated microcapsules and this effect was sustained at both glucose levels of 5.5 and $35.5 \mathrm{mM}$.

Conclusion: UDCA addition enhanced PB delivery and biological effects in a formulationdependent manner.

Keywords: probucol, microencapsulation, NM30D, ursodeoxycholic acid, diabetes, oxidative stress

\section{Introduction}

Diabetes mellitus (DM) is an epidemic and affects $8 \%$ of the world population and has two main types, Type-1 (T1D) and Type-2 (T2D) with T2D being the most dominant. DM prevalence has been rising rapidly worldwide. ${ }^{1}$ In Australia alone, one person is diagnosed with diabetes every 5 mins and this is costing the Australian health budget more than $\$ 10$ billion a year. ${ }^{2,3}$ Current treatment for T1D and T2D aim to control symptoms and reduce complications, but more than half of diabetic patients have uncontrolled diabetic symptoms despite strict adherence to diabetes therapy. ${ }^{4}$ The disease is a major cause of vision loss, heart attacks, kidney failure, stroke, and limb amputation. In 2016, 1.8 million deaths were directly attributed to DM, with the majority occurring before the age of 70 years. The World Health Organisation projects diabetes to be the seventh leading cause of
Biotechnology and Drug Development Research Laboratory, School of Pharmacy and Biomedical Sciences, Curtin Health Innovation Research Institute (CHIRI), Curtin University, Perth, WA, Australia Tel + 6I 892669816

$\mathrm{Fax}+61892662769$

Email hani.al-salami@curtin.edu.au 
death in 2030, unless new and far more robust and effective therapeutics are introduced to the market. ${ }^{5,6}$

DM-associated inflammation is seen throughout DM development and progression, and has been strongly associated with T2D complications such as heart disease and dyslipidaemia, although many patients may not exhibit strong clinical inflammation or severe macro/micro vascular complications. ${ }^{7}$ Obesity is a risk factor in $\mathrm{T} 2 \mathrm{D}$, and has been linked to increased morbidity and poor glycaemic control. In a study by Szpigel, et al; the authors showed that certain types of lipids in T2D can induce oxidative stress and inflammation, in mechanisms which are driven by immune cells. ${ }^{8}$ In another study by de Souza, et al; the authors showed that even in T2D patients with wellcontrolled symptoms, dyslipidaemia worsens diabetesassociated-inflammation and prognosis, which suggests direct link, in T2D, between inflammation, complications and glycaemic control. ${ }^{9}$ A study by $\mathrm{CY}$, et al has shown that lipids and oxidants are not only exacerbating inflammation, but also play an important role in initiating and promoting inflammation at the early stages of T2D. ${ }^{10}$ Thus, there is a need for new antidiabetic therapies, which are powerful, and can control symptoms, and alleviate diabetes-associated inflammation and complications. New treatments may include drugs with potent antioxidant, anti-inflammatory and lipid-lowering effects.

Probucol (PB) is an anti-atherosclerotic drug with potent antioxidant, anti-inflammatory and lipid-lowering effects. PB is taken orally and has poor solubility in water and variable gut absorption profile, which results in potentially severe side effects. Variation in gut absorption of $\mathrm{PB}$ can be due to variation in expression of gut proteins responsible for PB uptake, or the current formulations having unideal targeted delivery properties resulting in inconsistent uptake of PB and poor bioavailability. ${ }^{11}$ In one study, Rinninger, et al showed that specific protein transporters can have significant influence on the rate of PB cellular uptake. ${ }^{12}$ In another study, Ma, et al showed that the transporter $\mathrm{ABCB} 1$ exerted direct cellular and biological effects which significantly impacted $\mathrm{PB}$ cellular permeation, efficacy and safety profiles. ${ }^{13} \mathrm{~PB}$ targeted delivery is anticipated to optimise its absorption profile, and this can be achieved using the microencapsulation technology. ${ }^{14}$ Multiple studies in our laboratory have shown that sodium alginate (SA) polymer can be used to form microcapsules containing $\mathrm{PB}$, for targeted delivery of $\mathrm{PB}$, and incorporating bile acids into $\mathrm{PB}$ microcapsules can enhance further PB absorption and targeted delivery in the gut. ${ }^{15-20}$ However, to date, ideal SA-based microcapsules with robust targeted-delivery profiles have not been established, and thus, new polymers may need to be incorporated for better PB targeted delivery and cellular absorption. Eudragit polymers have good stability and have been proposed to enhance the targeted delivery and absorption of orally administered drugs. ${ }^{21-24}$ Accordingly, this study investigates the design and testing of new PB microcapsules incorporating bile acids and the polymer Eudragit, in terms of formulation stability, physical features, PB targeted release profile, and formulation effects on pancreatic $\beta$-cells. This study is part of our ongoing work on bile acids applications in drug delivery and diabetes therapy.

\section{Materials and Methods Reagents}

Alginate salt ( $99 \%$, medium viscosity with $65 \% \mathrm{M}$ to $\mathrm{G}$ ), probucol, ursodeoxycholic acid, and (3-(4,5-Dimethylthiazol2-yl)-2,5-Diphenyltetrazolium Bromide) (MTT) were all purchased from Sigma-Aldrich Corporation (Australia). Eudragit polymers were obtained from Evonik Industries (Victoria, Australia). 2',7'-dichlorofluorescin diacetate (DCFH-DA) was purchased from Sigma-Aldrich Corporation (New South Wales, Australia) and 2,2'-azobis-2-methyl-propanimidamide, dihydrochloride (AAPH) was purchased from Sapphire Bioscience (New South Wales, Australia).

\section{Pancreatic NIT-I $\beta$-Cells}

Cells were grown and processed in a Dulbecco's Modified Eagle's media with $11.5 \%$ foetal bovine serum, as per our protocols. $^{25,26}$

\section{Matrix Fabrication}

Formulations containing sodium alginate, a polymer: NM30D, RL30D or RS30D, and probucol were prepared without ursodeoxycholic acid (control) or with ursodeoxycholic acid (test). Microcapsules formed using our Ionic gelation vibrational jet flow technology, via Buchi-based system incorporating concentric nozzle connected with air supply, as per our established methods. ${ }^{19,27}$

\section{Morphological, Chemical, Thermal and Electrokinetic Stability Measurements}

Microcapsule morphology was analysed using scanning electron microscopy, chemical stability was analysed using Fourier Transformer Infra-Red spectroscopy, thermal stability was analysed using differential scanning 
calorimetry, and electrokinetic stability was analysed using Zeta-potential analyser, as per our established systems. ${ }^{18,28}$ In brief, morphology was analysed using Zeiss Neon 40EsB FIBSEM (USA) of freshly made microcapsules, which were coated and multiple images taken, while for chemical analysis, microcapsules were placed in PerkinElmer Spectrum 2 IR (PerkinElmer, USA) and analysed for chemical bond-formation spectrometry. For thermal analysis, melting points were measured for the different microcapsules using single furnace DSC 6000 instrumentation (PerkinElmer, USA) via isothermal analysis, while for electrokinetic stability, electrokinetic potential of the formulations were assessed using the Malvern Zetasizer $3000 \mathrm{HSa}$ (Malvern, UK), using $1 \mathrm{~mL}$ of microencapsulating formulations for each analysis, as per our well-established methodologies. ${ }^{29-31}$

\section{Microcapsule Physical and Mechanical Resistance, Osmotic Stability and Drug $\mathrm{pH}$-Release Measurements}

The mechanical resistance of microcapsules was assessed by measuring their physical ability to remain intact, under continuous shaking for 2 weeks at acidic and alkaline $\mathrm{pH}$ values of 1.5 and 7.4, using our established methods, while osmotic stability was measured using changes in water contents and thus weight of microcapsules over 2 weeks period, as per our well-established methods. ${ }^{32,33}$ Release of probucol from each microcapsule was measured as percentage of cumulative drug uptake by media surrounding the microcapsules, over $8 \mathrm{hrs}$ period at acidic and alkaline $\mathrm{pH}$ values (1.5 and 7.4). Probucol concentrations were measured using a spectrophotometer incorporated in a UV-dissolution instrument (PerkinElmer, USA), set at wavelength $241 \mathrm{~nm}$, as per our established methods. ${ }^{32,34}$

\section{Probucol Concentrations in NIT-I Cells, Effects on Cell Number (Viability) and Cellular Antioxidant Activity Measurements}

Intracellular uptake of probucol by NIT-1 cells was measured by incubating the microcapsules with the cells for 2 days, washing the cells to remove any media, rupturing them by sonication and analyzing intracellular contents for probucol concentrations. Intracellular uptake was measured at two different glucose concentrations, 5.5 and $35.5 \mathrm{mM}$ to assess uptake at normoglycemia and hyperglycaemia. High pressure liquid chromatography was used to measure probucol concentrations as per our established methods. ${ }^{35}$ Cell number (expressed as viability percentage) was assessed using cell number provided by MTT assays established in our laboratory, where microcapsules were incubated with NIT-1 cells for $48 \mathrm{hrs}$ prior to viability measurements. ${ }^{36}$ Antioxidant activity measurements were carried out at normoglycemia and hyperglycemia, for $48 \mathrm{hrs}$, using fluorescent activities through a mixture of dichloro-dihydro-fluorescein diacetate and 2,2'-azobis-2-methyl-propanimidamide, dihydrochloride, which were mixed with the cells and microcapsules. The fluorescent activities were measured using an Enspire Multimode Plate Reader (PerkinElmer, USA) at $485 \mathrm{~nm}$ absorption wavelength and $538 \mathrm{~nm}$ emission wavelength. Data were normalized for cell viability.

\section{Statistical Analysis}

Data are stated as means \pm standard error of the mean and statistical analysis was done using parametric/nonparametric or one-way ANOVA followed by Tukey posthoc as appropriate, using GraphPad Prism Version 7.04 (GraphPad, USA). Difference was reported when p-value $<0.05$ or $p$-value $<0.01$.

\section{Results and Discussion}

Microcapsule Morphology, Chemical and

\section{Thermal Stability}

The presence of different polymers (NM30D, RL30D or RS30D) or the bile acid ursodeoxycholic acid did not result in significant changes in the microcapsules' size, morphology, shape (Figure 1A), chemical (Figure 1B) and thermal (Figure 1C) compatibilities. Size was assessed using SEM scale bar and shape was determined using visual assessment of the micrographs, while chemical and thermal compatibilities were assessed by comparing test with control in terms of peaks. This suggests that microcapsules' constituents including probucol are compatible, and the encapsulating method is robust and consistent. Ursodeoxycholic acid incorporation with NM30D and RL30D polymers, in probucol formulation, enhanced Zeta-potential, with NM30D having the highest value of $94 \pm 3 \mathrm{mV}$ (Figure 1D). The increase in Zeta-potential suggests improved stability and such effects by ursodeoxycholic acid were formulation-dependent. This is in line with a previous study where ursodeoxycholic acid enhanced Zeta-potential of alginate-poly-l-ornithine formulation. ${ }^{37}$ Compatibility of microcapsules' constituents 


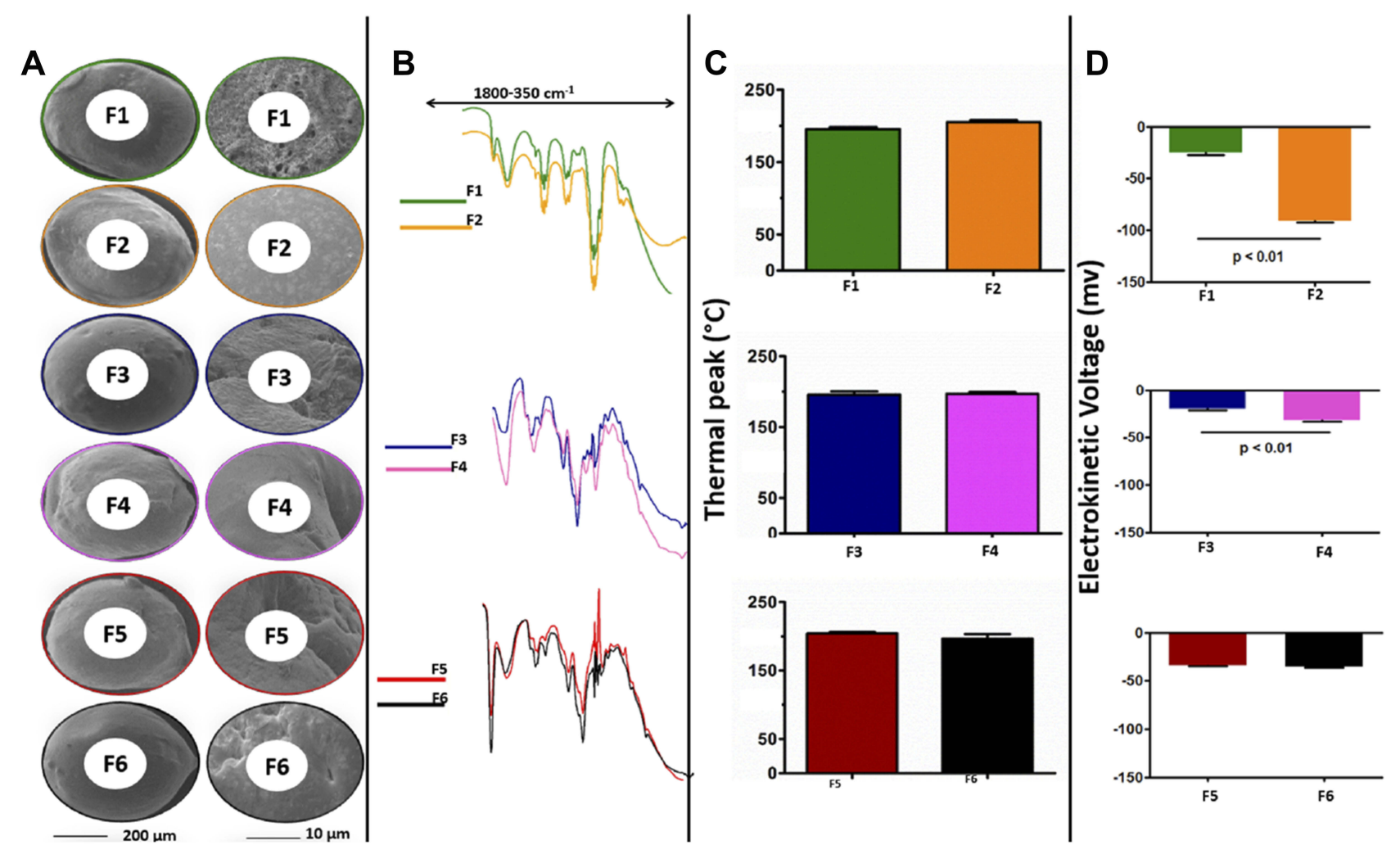

Figure I SEM images of microcapsule FI-F6 (A), FTIR-chromatograms (B), thermal peaks (C), and electrokinetic voltage (D). Data are mean \pm SEM, $\mathrm{n}=3$. F2, F4 and F6 are formulation matrices with UDCA, probucol and addition of NM30D, RL30D or RS30D polymers, respectively. FI, F3 and F5 are corresponding controls without UDCA.

and the high electrokinetic stability is likely to produce microcapsules with stable mechanical properties and consistent drug release profiles.

\section{Microcapsule Physical and Mechanical Resilience, Osmotic Stability and Drug $\mathrm{pH}-$ Release Measurements}

Figure 2 shows microcapsules' mechanical properties and resilience (Figure 2A), resistance to swelling and moisture loss (Figure 2B), and probucol release profiles (Figure 2C) at acidic and alkaline $\mathrm{pH}$ values of 1.5 and 7.4. Microcapsules resilience to mechanical and shear stress remained high regardless of the formulation or ursodeoxycholic acid incorporation, while water uptake and osmotic stability were enhanced by ursodeoxycholic acid incorporation only at alkaline $\mathrm{pH}$. Results suggest that all six formulations had sufficient structural integrity to sustain their shape, and remain intact, regardless of changes in $\mathrm{H}^{+}$concentrations and acidity of media, while the positive effects of ursodeoxycholic acid on osmotic stability are in line with previous studies showing membrane-stabilizing effects on alginate-based microcapsules. ${ }^{37,38}$ The three Eudragit polymers were chosen due to their wide solubility range and aqueous dispersion with NM30D being the only neutral copolymer with low viscosity, while RL30D having very high permeability and RS30D having very low permeability and widely used for its control release properties. The reason for osmotic stability being improved by ursodeoxycholic acid is possibly due to membrane-stabilizing effects, when combined with sodium alginate matrix and NM30D, RL30D or RS30D polymers. The consistently high mechanical resilience of the microcapsules is desirable, and mechanical strength is widely investigated in the literature. Chang, et al showed that incorporation of a unique shortchain alginate - co-MPEG biocompatible polymer into alginate-based microcapsules can significantly enhance mechanical strength and produce better delivery properties, ${ }^{39}$ while, Sun G and Zhang Z, compared mechanical strength of three different microcapsules made of different wall materials, and showed significant formulationdependent properties as a result of changes of membrane composition of microcapsules and subsequent changes to microcapsules' resilience when exposed to compression, deformation or rupture forces. ${ }^{40}$ Probucol release from microcapsules was negligible at the acidic $\mathrm{pH}$, likely due 

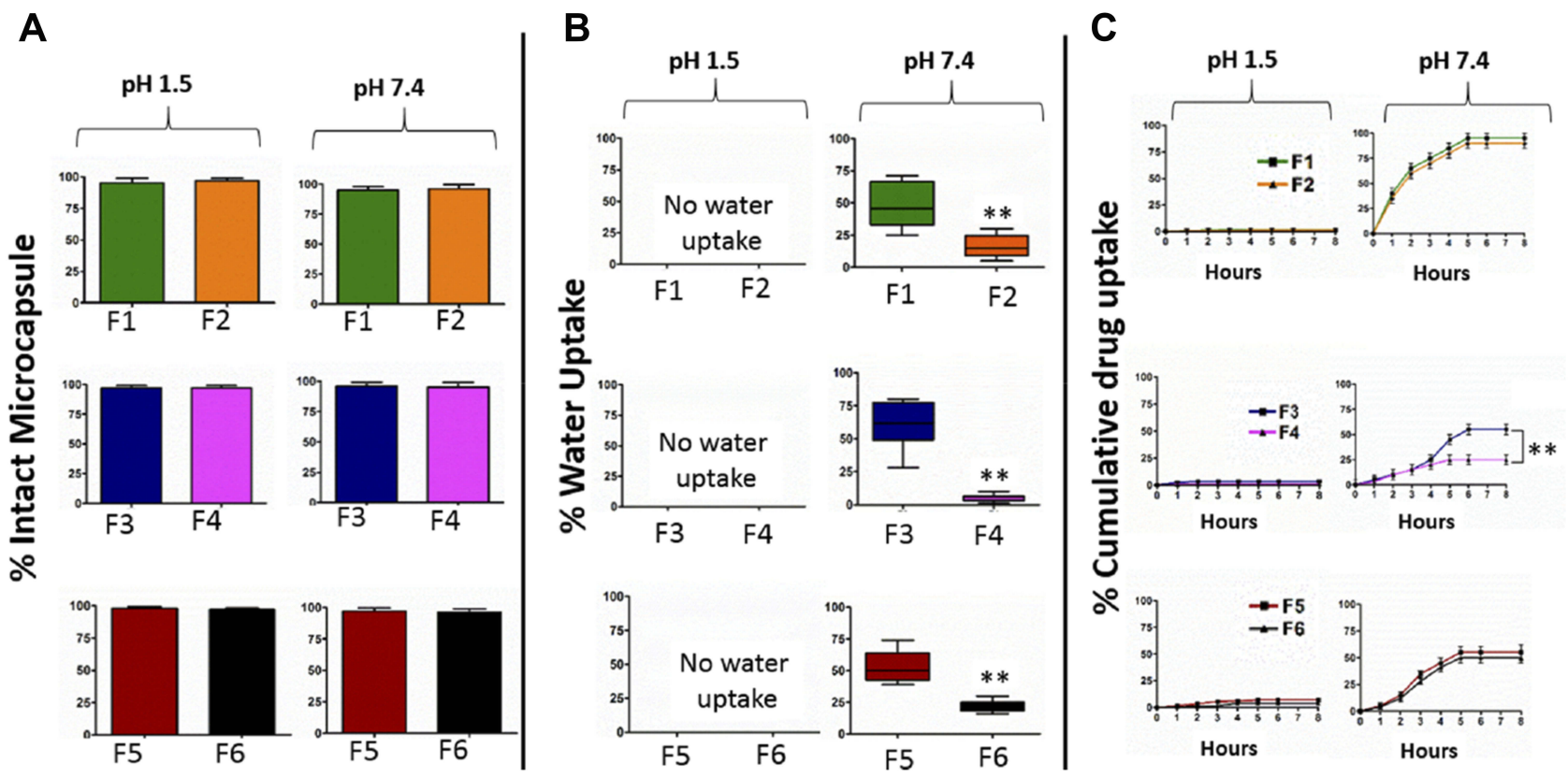

Figure 2 Mechanical stability $(\mathbf{A})$, osmotic stability $(\mathbf{B})$ and probucol release profile from the microcapsules at $\mathrm{pH} 1.5$ and 7.4 . Data are mean $\pm \mathrm{SEM}$, $\mathrm{n}=3$. $* * \mathrm{p}<1 \%$.

to the alginate maintaining its chemical integrity and shape, and resisting disintegration and drug release. This is consistent with osmotic stability of microcapsules where they showed no water uptake at the acidic $\mathrm{pH}$ (Figure 2B). Probucol release from microcapsules was significant at alkaline $\mathrm{pH}$, which suggests targeted-delivery properties at the lower part of the ileal mucosal, after oral administration of microcapsules. Probucol release at alkaline $\mathrm{pH}$ was low in RL30D microcapsules, and that was significantly lowered further by ursodeoxycholic acid, which suggests that RL30D polymer interacts with probucol or microcapsule's excipient(s) and prevents full microcapsule disintegration and probucol release, and ursodeoxycholic acid exacerbate this effect. Ursodeoxycholic acid exists in two physicochemical states, amorphous and crystalline. The crystalline state may reduce solubility of microcapsule's constituents including probucol, and this can result in lowering of probucol release from microcapsules due to slower disintegration and dissolution rates. ${ }^{41}$ Probucol release profile from the microcapsules at alkaline $\mathrm{pH}$ may influence probucol uptake by $\beta$-cells, and this was investigated in this paper (Figure 3).

\section{Probucol Cellular Uptake}

Probucol afflux into NIT-1 pancreatic cells was consistent with little variation of concentrations among all tested cells (Figure 3 ). There is a visible and strong intracellular uptake from the NM30D microcapsules incorporated with ursodeoxycholic acid (F2), which suggests that interaction between NM30D and ursodeoxycholic acid promotes probucol uptake by both improving cell mass and numbers, or by increasing amount of probucol accumulating in each cell. The positive anti-apoptotic effects of ursodeoxycholic acid are in line with the literature. In two published works, Rodrigues et al showed that ursodeoxycholic acid improved mass and function of hepatocytes, ${ }^{42,43}$ while Houten, et al have proposed that bile acids affect expression and functions of protein transporters located within cell membranes, and these transporters are responsible for drug uptake into the cells. $^{44}$ Since ursodeoxycholic acid improved probucol uptake only in the NM30D group, this effect seems to be formulation-dependent. In order to ascertain if increasing probucol intracellular uptake was due to increase in cell mass and numbers, cell viability and oxidative stress were measured (Figure 4). Oxidative stress could be used to assess cell survival, long term, and the effects of microcapsules on oxidative stress may correlate to their long-term effects on viability and functions.

\section{Cellular Viability and Oxidative Stress Activities}

At normoglycemic state (5.5 $\mathrm{mM}$ glucose), cell exposure to microcapsules did not have any significant effect on cell 


\section{NIT-1 $\beta$-cell Probucol Uptake}

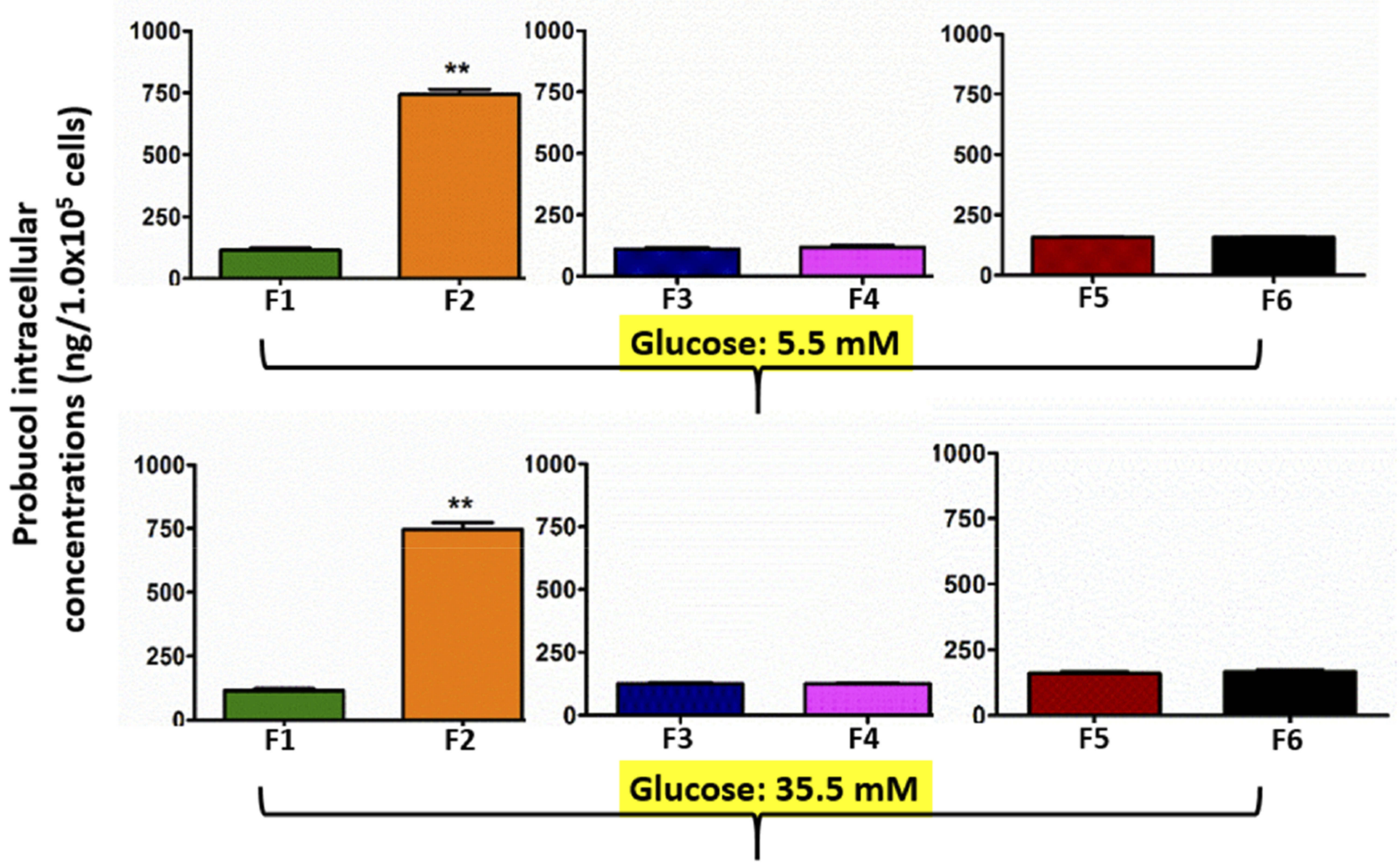

Figure 3 Pancreatic $\boldsymbol{\beta}$-cells uptake of probucol release from the microcapsules at glucose $5.5 \mathrm{mM}$ and $35.5 \mathrm{mM}$. Data are mean $\pm \mathrm{SEM}, \mathrm{n}=3$. ** $\mathrm{p}<1 \%$.



Figure 4 Viability of NIT-I cells after exposure to the microcapsules, and corresponding fluorescence (cellular oxidative stress) levels. Each treatment group (F2, F4 or F6) was compared with its corresponding control (FI, F3 or F5). Data are mean \pm SEM, n=3. *p-value $<0.05$, ** $p$-value $<1 \%$.

viability or oxidative stress, and all measured values remain the three control groups (F1, F3 and F5) showed similar cell very similar and with very little variations between measure- viability, and oxidative stress levels, and incorporation of ments (Figure 4). At hyperglycemic state $(35.5 \mathrm{mM}$ glucose), ursodeoxycholic acid enhanced cell viability among all 
microcapsules, but reduced oxidative stress only in the NM30D group (F2). Some of our previous studies ${ }^{45-47}$ suggest that UDCA in microcapsule formulation may exert a protective effect on pancreatic $\beta$-cells, desirable insulin production, and cell functionality and reduced inflammatory profile suggesting potential applications in diabetes. The positive effects of NM30D-ursodeoxycholic acid on cell viability and oxidative stress (Figure 4) may be associated with the positive effects observed on the electrokinetic voltage and Zeta-potential (Figure 1), and strong targetedrelease effects observed on microcapsules' release of probu$\mathrm{col}$ (Figure 2C) as well as the significant increase in probucol cellular-uptake observed (Figure 3 ). It is worth stating that cell exposure to probucol was enabled by its encapsulation, due to its lack of solubility in media.

Accordingly, findings of this paper indicate positive effects of NM30D-ursodeoxycholic acid incorporation into alginate-probucol microcapsules, in terms of microcapsules' stability, physical characteristics, drug release, drug cellular uptake, and cell viability and anti-oxidative stress effects, which suggest potential applications in probucol delivery and effects on pancreatic $\beta$-cells.

\section{Acknowledgments}

The authors acknowledge the use of laboratory equipment, and the scientific and technical assistance of the Microscopy and Microanalysis Facility at Curtin University, which has been partially funded by the University, State and Commonwealth Governments. AlSalami's work is partially supported by the European Union's Horizon 2020 research and innovation programme under the Marie Skłodowska-Curie grant agreement No 872370. The NIT-1 cells were a generous donation from Professor Grant Morahan at the University of Western Australia and were approved by Curtin.

\section{Author Contributions}

All authors contributed towards data analysis, drafting and critically revising the paper, gave final approval of the version to be published, and agreed to be accountable for all aspects of the work.

\section{Disclosure}

$\mathrm{H}$ Al-Salami has been, and is currently receiving, funding from Beijing Nat-Med Biotechnology Co., Ltd., and reports grants from EU Horizon 2020, outside the submitted work. The authors report no other conflicts of interest in this work.

\section{References}

1. LaVeist TA, Thorpe RJ Jr., Galarraga JE, Bower KM, Gary-Webb TL. Environmental and socio-economic factors as contributors to racial disparities in diabetes prevalence. J Gen Intern Med. 2009;24 (10):1144-1148. doi:10.1007/s11606-009-1085-7

2. Chittleborough CR, Grant JF, Phillips PJ, Taylor AW. The increasing prevalence of diabetes in South Australia: the relationship with population ageing and obesity. Public Health. 2007;121(2):92-99. doi:10.1016/j.puhe.2006.09.017

3. King H, Dixon J, Senator G, Zimmet P. Insulin-treated diabetes in Tasmania: population-based clinical characteristics and their possible implications for diabetic health care in Australia. Med J Aust. 1989;151(8):444, 446, 448, 450. doi:10.5694/j.1326-5377.1989. tb101254.x

4. D'Antonio JA, Ellis D, Doft BH, et al. Diabetes complications and glycemic control. The Pittsburgh prospective insulin-dependent diabetes cohort study status report after 5 yr of IDDM. Diabetes Care. 1989;12(10):694-700. doi:10.2337/diacare.12.10.694

5. Harris TJ, Cook DG, Wicks PD, Cappuccio FP. Impact of the new American Diabetes Association and World Health Organisation diagnostic criteria for diabetes on subjects from three ethnic groups living in the UK. Nutr Metab Cardiovasc Dis. 2000;10(6):305-309.

6. Alberti KG. The World Health Organisation and diabetes. Diabetologia. 1980;19(3):169-173. doi:10.1007/BF00275264

7. Polenova NV, Vaulin NA, Masenko VP, Iavelov IS, Gratsianskii NA. [Rosuvastatin and fenofibrate in patients with diabetes and low high density lipoprotein cholesterol: comparison of changes of lipid levels and some markers of inflammation]. Kardiologiia. 2009;49(2):9-14.

8. Szpigel A, Hainault I, Carlier A, et al. Lipid environment induces ER stress, TXNIP expression and inflammation in immune cells of individuals with type 2 diabetes. Diabetologia. 2017;61(2):399-412.

9. de Souza Bastos A, Graves DT, de Melo Loureiro AP, et al. Diabetes and increased lipid peroxidation are associated with systemic inflammation even in well-controlled patients. $J$ Diabetes Complications. 2016;30(8):1593-1599. doi:10.1016/j.jdiacomp.2016.07.011

10. Ha CY, Kim JY, Paik JK, et al. The association of specific metabolites of lipid metabolism with markers of oxidative stress, inflammation and arterial stiffness in men with newly diagnosed type 2 diabetes. Clin Endocrinol (Oxf). 2012;76(5):674-682. doi:10.1111/cen.2012.76.issue-5

11. Kubo Y, Terashima Y, Yagi N, Nochi H, Tamoto K, Sekikawa H. Enhanced bioavailability of probucol following the administration of solid dispersion systems of probucol-polyvinylpyrrolidone in rabbits. Biol Pharm Bull. 2009;32(11):1880-1884. doi:10.1248/bpb.32.1880

12. Rinninger F, Wang N, Ramakrishnan R, Jiang XC, Tall AR. Probucol enhances selective uptake of HDL-associated cholesteryl esters in vitro by a scavenger receptor B-I-dependent mechanism. Arterioscler Thromb Vasc Biol. 1999;19(5):1325-1332. doi:10.1161/01.ATV.19.5.1325

13. Ma Q, Han Y, Chen C, et al. Oral absorption enhancement of probucol by PEGylated G5 PAMAM dendrimer modified nanoliposomes. Mol Pharm. 2015;12(3):665-674. doi:10.1021/mp500388m

14. Mooranian A, Negrulj R, Chen-Tan N, et al. Microencapsulation as a novel delivery method for the potential antidiabetic drug, Probucol. Drug Des Devel Ther. 2014;8:1221-1230. doi:10.2147/DDDT. S67349

15. Mooranian A, Negrulj R, Al-Sallami HS, et al. Probucol release from novel multicompartmental microcapsules for the oral targeted delivery in type 2 diabetes. AAPS PharmSciTech. 2015;16(1):45-52. doi:10.1208/s12249-014-0205-9

16. Mooranian A, Negrulj R, Arfuso F, Al-Salami H. The effect of a tertiary bile acid, taurocholic acid, on the morphology and physical characteristics of microencapsulated probucol: potential applications in diabetes: a characterization study. Drug Deliv Transl Res. 2015;5(5):511-522. doi:10.1007/s13346-0150248-9 
17. Mooranian A, Negrulj R, Arfuso F, Al-Salami H. Multicompartmental, multilayered probucol microcapsules for diabetes mellitus: formulation characterization and effects on production of insulin and inflammation in a pancreatic beta-cell line. Artif Cells Nanomed Biotechnol. 2016;44 (7):1642-1653. doi:10.3109/21691401.2015.1069299

18. Mooranian A, Negrulj R, Chen-Tan N, Watts GF, Arfuso F, Al-Salami H. An optimized probucol microencapsulated formulation integrating a secondary bile acid (deoxycholic acid) as a permeation enhancer. Drug Des Devel Ther. 2014;8:1673-1683. doi:10.2147/DDDT.S68247

19. Mooranian A, Negrulj R, Al-Sallami HS, et al. Release and swelling studies of an innovative antidiabetic-bile acid microencapsulated formulation, as a novel targeted therapy for diabetes treatment. JMicroencapsul. 2015;32(2):151-156. doi:10.3109/02652048.2014.958204

20. Negrulj R, Mooranian A, Chen-Tan N, et al. Swelling, mechanical strength, and release properties of probucol microcapsules with and without a bile acid, and their potential oral delivery in diabetes. Artif Cells Nanomed Biotechnol. 2016;44(5):1290-1297. doi:10.3109/ 21691401.2015.1024845

21. Cetin M, Atila A, Sahin S, Vural I. Preparation and characterization of metformin hydrochloride loaded-Eudragit ${ }^{\mathbb{B}}$ RSPO and Eudragit ${ }^{\mathbb{R}}$ RSPO/PLGA nanoparticles. Pharm Dev Technol. 2013;18 (3):570-576. doi:10.3109/10837450.2011.604783

22. Enose Appavoo A, Anand P, Bhaskar K, Ramachandran S, Saravanan M, Vinod R. Eudragit NE30D based metformin/gliclazide extended release tablets: formulation, characterisation and in vitro release studies. Notes. 2002;50(11):1495-1498.

23. Devarajan PV, Sonavane GS. Preparation and in vitro/in vivo evaluation of gliclazide loaded Eudragit nanoparticles as a sustained release carriers. Drug Dev Ind Pharm. 2007;33(2):101-111. doi:10.1080/03639040 601096695

24. Arno EA, Anand P, Bhaskar K, Ramachandran S, Saravanan M, Vinod R. Eudragit NE30D based metformin/gliclazide extended release tablets: formulation, characterisation and in vitro release studies. Chem Pharm Bull (Tokyo). 2002;50(11):1495-1498. doi:10.1248/cpb.50.1495

25. Mooranian A, Tackechi R, Jamieson E, Morahan G, Al-Salami H. Innovative microcapsules for pancreatic beta-cells harvested from mature double-transgenic mice: cell imaging, viability, induced glucosestimulated insulin measurements and proinflammatory cytokines analysis. Pharm Res. 2017;34(6):1217-1223. doi:10.1007/s11095-0172138-y

26. Mooranian A, Negrulj R, Takechi R, Jamieson E, Morahan G, Al-Salami H. Electrokinetic potential-stabilization by bile acid-microencapsulating formulation of pancreatic beta-cells cultured in high ratio poly-L-ornithine-gel hydrogel colloidal dispersion: applications in cell-biomaterials, tissue engineering and biotechnological applications. Artif Cells Nanomed Biotechnol. 2018;46(6):1156-1162.

27. Mooranian A, Negrulj R, Mathavan S, et al. Stability and release kinetics of an advanced gliclazide-cholic acid formulation: the use of artificial-cell microencapsulation in slow release targeted oral delivery of antidiabetics. J Pharm Innov. 2014;9(2):150-157. doi:10.1007/s12247-014-9182-5

28. Mooranian A, Negrulj R, Mathavan S, et al. An advanced microencapsulated system: a platform for optimized oral delivery of antidiabetic drug-bile acid formulations. Pharm Dev Technol. 2015;20;6:702-709.

29. Mooranian A, Negrulj R, Arfuso F, Al-Salami H. The effect of a tertiary bile acid, taurocholic acid, on the morphology and physical characteristics of microencapsulated probucol: potential applications in diabetes: a characterization study. Drug Deliv Transl Res. 2015;5:1-12.

30. Mooranian A, Negrulj R, Mikov M, Golocorbin-Kon S, Arfuso F, AlSalami H. Novel chenodeoxycholic acid-sodium alginate matrix in the microencapsulation of the potential antidiabetic drug, probucol. An in vitro Study. J Microencapsulation. 2015;32:1-9.

31. Negrulj R, Mooranian A, Chen-Tan N, et al. Swelling, mechanical strength, and release properties of probucol microcapsules with and without a bile acid, and their potential oral delivery in diabetes. Artif Cells Nanomed Biotechnol. 2015;44:1290-1297.
32. Mooranian A, Negrulj R, Al-Sallami HS, et al. Release and swelling studies of an innovative antidiabetic-bile acid microencapsulated formulation, as a novel targeted therapy for diabetes treatment. J Microencapsul. 2014;32:1-6.

33. Mooranian A, Negrulj R, Mathavan S, et al. Stability and release kinetics of an advanced gliclazide-cholic acid formulation: the use of artificial-cell microencapsulation in slow release targeted oral delivery of antidiabetics. $J$ Pharm Innov. 2014;9:1-8.

34. Mooranian A, Negrulj R, Al-Sallami HS, et al. Probucol release from novel multicompartmental microcapsules for the oral targeted delivery in type 2 diabetes. AAPS PharmSciTech. 2014;16:45-52.

35. Mooranian A, Negrulj R, Takechi R, Mamo JC, Al-Sallami H, AlSalami H. The biological effects of the hypolipidaemic drug probucol incorporated into bile acid-microcapsules and fed daily for 4-weeks, to an insulin-resistant mouse model: potential hypoglycaemic and anti-inflammatory effects. Drug Deliv Transl Res. 2018;8:543-551. doi:10.1007/s13346-017-0473-5

36. Mooranian A, Negrulj R, Jamieson E, Morahan G, Al-Salami H. Biological assessments of encapsulated pancreatic $\beta$-cells: their potential transplantation in diabetes. Cell Mol Bioeng. 2017;9:530-537.

37. Mooranian A, Negrulj R, Chen-Tan N, et al. Advanced bile acid-based multi-compartmental microencapsulated pancreatic beta-cells integrating a polyelectrolyte-bile acid formulation, for diabetes treatment. Artif Cells Nanomed Biotechnol. 2016;44 (2):588-595. doi:10.3109/21691401.2014.971806

38. Mooranian A, Negrulj R, Arfuso F, Al-Salami H. Characterization of a novel bile acid-based delivery platform for microencapsulated pancreatic beta-cells. Artif Cells Nanomed Biotechnol. 2016;44 (1):194-200. doi:10.3109/21691401.2014.934457

39. Chang SJ, Lee CH, Hsu CY, Wang YJ. Biocompatible microcapsules with enhanced mechanical strength. J Biomed Mater Res. 2002;59 (1):118-126. doi:10.1002/(ISSN)1097-4636

40. Sun G, Zhang Z. Mechanical strength of microcapsules made of different wall materials. Int $J$ Pharm. 2002;242(1-2):307-311. doi:10.1016/S0378-5173(02)00193-X

41. Okonogi S, Oguchi T, Yonemochi E, Puttipipatkhachorn S, Yamamoto K. Physicochemical properties of ursodeoxycholic acid dispersed in controlled pore glass. J Colloid Interface Sci. 1999;216 (2):276-284. doi:10.1006/jcis.1999.6331

42. Rodrigues CM, Fan G, Ma X, Kren BT, Steer CJ. A novel role for ursodeoxycholic acid in inhibiting apoptosis by modulating mitochondrial membrane perturbation. J Clin Invest. 1998;101(12):27 90-2799. doi:10.1172/JCI1325

43. Rodrigues C, Fan G, Wong PY, Kren BT, Steer CJ. Ursodeoxycholic acid may inhibit deoxycholic acid-induced apoptosis by modulating mitochondrial transmembrane potential and reactive oxygen species production. Mol Med. 1998;4(3):165. doi:10.1007/BF03401914

44. Houten SM, Watanabe M, Auwerx J. Endocrine functions of bile acids. EMBO J. 2006;25(7):1419-1425. doi:10.1038/sj.emboj.7601049

45. Mooranian A, Negrulj R, Arfuso F, Al-Salami H. Characterization of a novel bile acid-based delivery platform for microencapsulated pancreatic $\beta$-cells. Artif Nanomed Biotechnol. 2016;44(1):194-200. doi:10.3109/21691401.2014.934457

46. Mooranian A, Negrulj R, Al-Salami H. The incorporation of watersoluble gel matrix into bile acid-based microcapsules for the delivery of viable $\beta$-cells of the pancreas, in diabetes treatment: biocompatibility and functionality studies. Drug Deliv Transl Res. 2016;6 (1):17-23. doi:10.1007/s13346-015-0268-5

47. Mooranian A, Zamani N, Takechi R, et al. Pharmacological Effects of Nanoencapsulation of Human-Based Dosing of Probucol on Ratio of Secondary to Primary Bile Acids in Gut, During Induction and Progression of Type 1 Diabetes. Artif Cells Nanomed Biotechnol. 2018;46(sup3):S748-S754. 


\section{Publish your work in this journal}

Nanotechnology, Science and Applications is an international, peerreviewed, open access journal that focuses on the science of nanotechnology in a wide range of industrial and academic applications. It is characterized by the rapid reporting across all sectors, including engineering, optics, bio-medicine, cosmetics, textiles, resource sustainability and science. Applied research into nano-materials, particles, nano-structures and fabrication, diagnostics and analytics, drug delivery and toxicology constitute the primary direction of the journal. The manuscript management system is completely online and includes a very quick and fair peer-review system, which is all easy to use. Visit http://www.dovepress.com/testimonials.php to read real quotes from published authors. 